\title{
Can infections cause Alzheimer's disease?
}

\author{
Elliott Asher and Satesh Bidaisee* \\ Public Health and Preventive Medicine, St. George's University, University Centre, Grenada
}

\begin{abstract}
This review explores the possibility of an infectious etiology for Alzheimer's disease (AD) as the body of evidence to support an infectious cause is increasing. It is becoming very important from public health perspective as the number of individuals reaching and passing the age of 65 is increasing globally and due to lack of a solution for this disease it is costing the society a great deal of financial burden. AD is the most common form of dementia with a typical late-onset. While obvious tangles and threads are observed in the brain of patients with $\mathrm{AD}$, inflammation is now becoming another reason associated with $\mathrm{AD}$. Inflammation could be caused by central nervous system infection. A range of bacterial or viral agents, such as C. pneumonia, or herpes simplex type I, respectively, in turn, could cause the inflammation. Other agents such as prion have been hypothesized to play a role in the genesis of AD. In order to effectively diagnose and treat $\mathrm{AD}$, it is paramount to solve and identify the etiological and the source that contribute to the formation of this disease either by infectious diseases or other factors. Alzheimer's Disease, (also known as AD, Senile Dementia of the Alzheimer Type (SDAT) or simply Alzheimer's), is defined clinically as a neurological disorder that leads the brain cells to stop functioning and die, causeing loss of memory and a decline in the cognitive pathways. AD is a form of dementia, a neurodegenerative type, which starts slow and mild and progressively worsens over a period of time.
\end{abstract}

\section{Introduction}

Alzheimer's disease is currently an incurable, degenerative, terminal disease was first described by a German psychiatrist and neuropathologist in 1906 - Alois Alzheimer._Alzheimer's, as a slowly progressive disease of the brain, is characterized by impairment of memory and eventually by disturbances in reasoning, planning, language, and perception. It is believed that Alzheimer's results from an increase in the production or accumulation of a specific protein (beta-amyloid protein) in the brain that leads to nerve cell death.

Alzheimer's is an irreversible, progressive brain disorder that eventually impairs the ability for the person to carry out the simplest tasks. In most Alzheimer's patients, symptoms first appear in their mid-60s. Estimates vary, but experts suggest that more than 5 million Americans may have Alzheimer's. Generally, Alzheimer's is diagnosed in people over 65 years of age, although the less-prevalent early onset of Alzheimer's can occur much earlier. Alzheimer's is predicted to affect 1 in 85 people globally by the year 2050. Alzheimer's is diagnosed according to the guidelines recommended by the Diagnostic and Statistical Manual of Mental Disorders, Fifth Edition and the National Institute of Neurological Disorders and Stroke-Alzheimer Disease and Related Disorders working group [1]. Although these guides provide ways to detect and diagnose Alzheimer's, the most certain and definitive way to know if Alzheimer's is present is through histopathological examination of either biopsy or postmortem brain tissue. In most cases, the individual affected by Alzheimer's exhibits gradual memory degeneration and predominant cognitive deficit in the early stage, while as the disease progresses towards the more advanced stages, other cognitive deficits, such as motor and sensory deficits, personality changes and behavioral disorders become more apparent [2].

As Alzheimer's progresses, the signs become more pronounced and symptoms become more frequent until the person affected by it is completely disabled, which eventually leads to death. Signs include delirium, which has an acute onset, with an altered state of consciousness (treatable); depression with a gradual onset, showing feelings of low self-esteem (treatable); and then dementia with gradual onset, showing memory loss and decline in cognitive function (possibly treatable). The symptoms typically include: sleeplessness, wandering and pacing, aggression, agitation, anger, delusions, depression and hallucinations [2].

\section{Categories of AD}

Alzheimer's is clinically categorized into early onset, which is uncommon and occurs in ages less than 65 and constitute about less than $1 \%$ of all Alzheimer's diagnosed cases and is caused primarily by autosomal dominant mutations in either the presenilin genes or the amyloid precursor protein [3]. Causes at the late onset are unknown for the most part, but some risk factors that can contribute to this later onset have been identified and include: trauma to the head, diabetes mellitus, hypertension, certain genetic variants and increasing age [4-6].

Early stage: The late onset, which is the most common, is further categorized into several stages: The first stage is the Early Stage. This stage is considered as a mild/early stage and the duration period is 2-4 years. Signs at the Early Stage include frequent recent memory loss of recent conversations and events, repeated questions, some problems expressing and understanding language, difficulty writing and using objects, depression and apathy. Also apparent are evidence of drastic personality changes with functional decline, need to set reminders for daily activities, and difficulties with sequencing impact driving early in this stage.

Correspondence to: Satesh Bidaisee, Public Health and Preventive Medicine, St George's University, University Centre, Grenada, Tel: (473) 439-2000; Fax: (473) 444-1219; Email: sbidaisee@sgu.edu

Key words: alzheimers disease, infections, dementia, neurodegeneration, chronic diseases

Received: February 18, 2017; Accepted: March 07, 2017; Published: March 10, 2017 
Moderate stage: The Moderate Stage is considered as a middle/ moderate stage and the duration is 2-10 years. In this stage, the person can no longer cover up problems; pervasive and persistent memory loss impacts life across settings; rambling speech, unusual reasoning, confusion about current events, time, and place are evident; increased potential to become lost in familiar settings, sleep disturbances, and mood or behavioral symptoms accelerate. Nearly $80 \%$ of patients exhibit emotional and behavioral problems, which are aggravated by stress and change. Slowness, rigidity, tremors, and gait problems impact mobility and coordination. Important at this stage is having a structure, reminders, and assistance with activities of daily living. During this stage, increased memory loss and confusion, problems recognizing family and friends, inability to learn new things, difficulty carrying out tasks that involve multiple steps (such as getting dressed), problems coping with new situations, delusions and paranoia, and impulsive behavior become the normal routine. In moderate Alzheimer's, damage occurs in areas of the brain that control language, reasoning, sensory processing, and conscious thought.

Late stage: The Late Stage is considered the most severe stage and the duration is 1-3 years. The Alzheimer's patient gets confused about the past and the present, no longer recognizes familiar people and places, generally is incapacitated with severe to total loss of verbal skills, is unable to care for self, risks the possibility of falling, is generally immobile most of the time, suffers problems with swallowing, incontinence, and illness, experiences extreme mood swings, behavioral problems, hallucinations, and delirium. At this stage, Alzheimer's patients need total support and care, and often die from infections or pneumonia.

\section{Diagnosis of Alzheimer's}

Alzheimer's is usually diagnosed clinically from the patient history, collateral history from relatives, and clinical observations, based on the presence of characteristic neurological and neuropsychological features and the absence of alternative conditions. Advanced medical imaging with computed tomography (CT) or magnetic resonance imaging (MRI), and with single photon emission computer tomography (SPECT) or positron emission tomography (PET) can be used to help exclude other cerebral pathology or subtypes of dementia. The diagnosis can be confirmed with very high accuracy post-mortem when brain material is available and can be examined histologically.

Neuropsychological tests such as the mini-mental state examination (MMSE) are widely used to evaluate the cognitive impairments needed for diagnosis. More comprehensive test arrays are necessary for high reliability of results, particularly in the earliest stages of the disease.

Psychological tests for depression are employed, since depression can either be concurrent with Alzheimer's, an early sign of cognitive impairment, or even the cause.

When available as a diagnostic tool, SPECT and PET neuroimaging are used to confirm a diagnosis of Alzheimer's in conjunction with evaluations involving mental status examination. In a person already suffering from dementia, SPECT appears to be superior in differentiating Alzheimer's from other possible causes, compared with the usual attempts employing mental testing and medical history analysis.

\section{Infectious diseases}

Causes of Alzheimer's are not yet fully understood, but it is clear that it develops because of a complex series of events that take place in the brain over a long period of time. It is likely that the causes include genetic, environmental, and lifestyle factors. Some drug therapies propose that Alzheimer's is caused by reduced synthesis of the neurotransmitter acetylcholine. Other cholinergic effects have also been proposed, such as initiation of large-scale aggregation of amyloid leading to generalized neuroinflammation.

Alzheimer's is characterized by a build-up of proteins in the brain. Though this cannot be measured in a living person, extensive autopsy studies have revealed this phenomenon. The build-up manifests in two ways: Plaques, which are deposits of the protein beta-amyloid that accumulate in the spaces between nerve cells; and Tangles, which are deposits of the protein tau that accumulate inside the nerve cells.

Alzheimer's is also characterized by loss of neurons and synapses in the cerebral cortex and certain subcortical regions. This loss results in gross atrophy of the affected regions, including degeneration in the temporal lobe and parietal lobe, and parts of the frontal cortex and cingulate gyrus. Both amyloid plaques and neurofibrillary tangles are clearly visible by microscopy in brains of those afflicted by AD. Plaques are dense, mostly insoluble deposits of amyloid - beta peptides and cellular material outside and around neurons.

Tangles (neurofibrillary tangles) are aggregates of the microtubuleassociated protein tau, which has become hyperphosphorylated and accumulate inside the cells themselves.

Although many older individuals develop some plaques and tangles as a consequence of ageing, the brains of $\mathrm{AD}$ patients have a greater number of them in specific brain regions such as the temporal lobe.

Alzheimer's disease has been identified as a protein misfolding disease (proteopathy), caused by accumulation of abnormally folded beta-amyloid (also written as A-beta or $A \beta$ ) and tau proteins in the brain. Plaques are made up of small peptides, 39-43 amino acids in length, $A \beta$.

Beta-amyloid is a fragment from a larger protein called amyloid precursor protein (APP), a transmembrane protein that penetrates through the neuron's membrane. APP is critical to neuron growth, survival and post-injury repair. In Alzheimer's disease, an unknown process causes APP to be divided into smaller fragments by enzymes through proteolysis.

One of these fragments gives rise to fibrils of beta-amyloid, which form clumps that deposit outside neurons in dense formations known as senile plaques.

$\mathrm{AD}$ is also considered a tauopathy due to abnormal aggregation of the tau protein. Every neuron has a cytoskeleton, an internal support structure partly made up of structures called microtubules.

These microtubules act like tracks, guiding nutrients and molecules from the body of the cell to the ends of the axon and back. A protein called tau stabilizes the microtubules when phosphorylated, and is therefore called a microtubule-associated protein.

In $\mathrm{AD}$, tau undergoes chemical changes, becoming hyperphosphorylated; it then begins to pair with other threads, creating neurofibrillary tangles and disintegrating the neuron's transport system.

\section{Can infectious disease cause AD?}

While a great deal of knowledge has been accumulated regarding plaques, tangles, $A \beta$, tau, and APP, in recent years, a serious attention has 
been given to the possibility that $\mathrm{AD}$ could also be caused by infectious diseases. It is known that infectious agents can lead to progressive and chronic central nervous system diseases and syndromes.

Research has shown neurocognitive decrease in humans and experimental animals in the presence of some of the infectious agents namely Chlamydophila pneumonia, Borrelia burgdorferi, Helicobacter pylori and herpes simplex virus type 1 (HSV-1) [7]. Therefore, these agents are suspected to be possible causes of $\mathrm{AD}$. If CNS is infected directly causing neuroinflammation, or indirectly through different effects of systemic inflammation on the brain, then these agents could induce $\mathrm{AD}$.

$\mathrm{AD}$ could also be induced by these infectious pathogens that cause an autoimmune response which targets the brain which in turn cause neuroinflammation [8]. It should be noted however that so far, no specific pathogen has been linked to the late onset of $\mathrm{AD}$ in humans.

\section{Compatibility of AD pathology with infections}

Neurohistopathology of Alzheimer's disease indicates presence of neuronal degeneration, tangles (intraneuronal neurofibrillary), threads (neuropil) and plaques (extracellular $\beta$-amyloid $(\mathrm{A} \beta)$ ) in a specific area and region of the brain. Tangles and threads are inside nerve cell bodies and dendritic processes and are formed due to hyperphosphorylation of tau fibers, which accumulate into fibrils that is insoluble [9]. Plaques on the other hand, accumulate outside of the cell bodies as a result of $\mathrm{A} \beta$ peptides [10].

The point to be made in here is that while the simultaneous presence of plaques, tangles and the threads is the histopathologic hallmark of Alzheimer's disease, they also occur in different CNS conditions such as chronic infections: $A \beta$ plaques are seen in prion protein diseases like CJD (Creutzfeldt-Jakob disease) and Kuru, and threads and tangles are seen in "measles virus-induced subacute sclerosing panencephalitis" and in syphilis. During acute and chronic infections, such as tuberculosis and leprosy, we frequently see an elevated level of amyloid accumulation in the tissues [11]. Some studies have shown that $A \beta$ has antimicrobial properties that when exposed to infection, the $\mathrm{A} \beta$ level rises, supporting the idea that its production in $\mathrm{AD}$ may be induced by infectious pathogens.

Microglia, astrocytes and cytokines are inflammatory components that are seen in significantly high levels in $\mathrm{AD}$ neuropathology. While inflammation is not just triggered by infectious pathogens, it is still typical of infectious diseases, therefore it is hypothesized that Alzheimer's disease could be due to an infection [12]. An alternative hypothesis states that neuroinflammation in $\mathrm{AD}$ is a reflection of an autoimmune response where the infectious pathogens trigger antibodies production which attack the brain [13]. These antibodies have been found in patients with $\mathrm{AD}$, however, to this date, no pathogen has been linked to the antibody production in $\mathrm{AD}[14]$.

\section{Neurotropic infectious agents that are suspects in caus- ing AD}

The following neurotropic agents may directly infect the brain and later cause the formation of $\mathrm{AD}$ :

Herpes Simplex Virus Type-I (HSV-1): Herpes simplex virus type-1 (HSV-1) is suspected of causing AD. As age increases, the serum level of HSV-1 increases and its level is highest in individuals of 60 years and older. Similarly, the number of individuals inflicted with $\mathrm{AD}$ also increases at age 65 and older $[15,16]$.
C. Penumoniae: C. pneumoniae is a bacterium that through droplet inhalation is spread from person to person and is associated with pneumonia and bronchitis that are lower-respiratory-tract diseases. It has been found both directly and indirectly in postmortem AD brain tissue. As a result, C. pneumoniae is also hypothesized to be involved in the formation of $\mathrm{AD}$ [17].

B. Burgdorferi: B. burgdorferi is a bacterium that through the bite of an infected tick is transmitted from vertebrate hosts to humans. Through examination of postmortem samples of brain tissue of the $\mathrm{AD}$ patients, $\mathrm{B}$. burgdorferi has been directly detected and isolated, suggesting that there may be associations between this bacterium and formation of Alzheimer's disease [18].

H. Pylori: H. pylori are Gram-negative bacteria that are associated with the upper gastrointestinal disorders such as ulcer, and gastric cancer. During Mini-Mental State Examination, Patients with AD showed a severe cognitive impairment when infected with $H$. pylori, compared to those that were not infected. It is also hypothesized that there is an association between H. pylori and Alzheimer's disease [19].

\section{Systemic infectious agents that could cause AD}

Systemic infections are can also play a role in the genesis of AD. For example, it has been shown that cognitive impairment and neurodegeneration is worse in children who survived sepsis compared to normal children. It is therefore hypothesized that chronic systemic infections can cause damage to the CNS leading to $\mathrm{AD}$ [20].

Conversely, it is suggested that individual with Alzheimer's disease, have more incidences of systemic infections which, supports the same hypothesis as described above. For example, more people have been admitted to the hospital with system infections such as pneumonia and UTI that already have AD than compared to those admitted without the $\mathrm{AD}[21]$.

Prions are the misshapen proteins that replicate by inducing normal proteins to misfold and aggregate in the brain, leading to several rare and chronic neurodegenerative disorders in humans, including Creutzfeldt-Jakob disease (CJD), variant Creutzfeldt-Jakob disease, Gerstmann-Sträussler-Scheinker syndrome. Prions enter the body through certain medical procedures involving the eyes or brain. In recent years, it has been discovered that similar processes of protein misfolding are at work in many neurodegenerative disorders, including Alzheimer's, Parkinson's and Lou Gehrig's disease [22].

In 2015, Nature published an article about the transmissibility of misfolded proteins prions between humans that underlie the pathology of Alzheimer's disease. Between 1958 and 1985, a number of individuals with short stature received shots of human growth hormone extracted from the pituitary glands of cadavers. Some of these samples were contaminated with prions that caused some patients to develop Creutzfeldt-Jakob disease.

To explore the question of human transmission, neuroscientists at University College London, conducted an autopsy study of eight patients who died from CJD after treatment with cadaver-derived growth factor. The researchers examined the autopsies of eight patients with CJD, aged 36 to 51, typically too young to exhibit Alzheimer's pathology who had all received injection of human growth hormone from cadavers in childhood. To their surprise, they found that six of the brains had the amyloid-beta pathology found in Alzheimer's patients, and four exhibited some degree of cerebral amyloid angiopathy, in which amyloid deposits build up on the walls of blood vessels in 
the brain. In those four, they found moderate to severe amount of gray matter and vascular amyloids plaques in their brains. Both gray matter and amyloid plaques are hallmarks of Alzheimer's disease. The researchers did not find a genetic mutation associated with early-onset Alzheimer's disease in these individuals. Additionally, they didn't observe an accumulation of tau proteins, which are another indicator of Alzheimer's disease.

The researchers concluded that while these patients hadn't yet developed symptoms of Alzheimer's disease, they most likely would have later in life. The findings suggested the human growth hormone the patients received in childhood contained the "seeds" of amyloid plaques, in addition to prions. As of 2012, researchers have identified 450 cases of CJD worldwide that are the result of these growth hormone injections and other medical procedures, including neurosurgery and transplants.

In this paper, Alzheimer's disease was discussed along with its genesis due to potentially different infectious agents involved. At this point there is no cure for $\mathrm{AD}$, however, treatments primarily focus on the support of the $\mathrm{AD}$ patients as they progress through this disease, they would need more to be taken care of by the family member or nursing staff.

So far no specific factor has been found to cause $\mathrm{AD}$, but there seems to be a multitude of agents that may paly a role in $\mathrm{AD}$ formation. Some neurotropic infectious agents such as HSV-1, C. pneumoniae and $\mathrm{H}$. pylori are suspected to play a role in changing the brain the way it functions, leading to the genesis of AD. There also seems to be an involvement of systemic infections and the formation of $\mathrm{AD}$ in the case of children with sepsis.

More work should be done to determine the role of antibiotic and Alzheimer's to see if its impact is due to CNS pathogen, other AD pathogens. Transmission of infectious agents between humans is seen in a study done on CJD victims who were all injected with the same growth hormone extracted from the pituitary glad of cadavers.

\section{Conclusion}

In conclusion, while the specific reason and cause of the $\mathrm{AD}$ and the role of infections is not known, there has been a growing body of evidence showing association between $\mathrm{AD}$ and infections. More research should be done to see if $\mathrm{AD}$ is preceded by CNS infection or to establish whether patients are at higher risk due to systemic infections and to finally see if indeed the infection transmission is possible between humans. Only finding answers to these questions will eventually enable us to diagnose, treat, and cure this disease, managing and freeing the society from the resource and financial burden that it is inflicting upon globally.

\section{References}

1. McKhann GM, Knopman DS, Chertkow H, Hyman BT, Jack CR, et al. (2011) The diagnosis of dementia due to Alzheimer's disease: Recommendations from the National Institute on Aging-Alzheimer's Association workgroup. Alzheimer's \& dementia 7: 263-269.

2. McKhann G, Drachman D, Folstein M, Katzman R, Price D, Stadlan EM, 1984. Clinical diagnosis of Alzheimer's disease: report of the NINCDS-ADRDA Work Group under the auspices of Department of Health and Human Services Task Force on Alzheimer's Disease. Neurology 34: 939-944.

3. Antonell A, Balasa M, Oliva R, Lladó A, Bosch B, et al. (2011) A novel PSEN1 gene mutation (L235R) associated with familial early-onset Alzheimer's disease. Neurosci Lett 496: 40-42.[Crossref]

4. Coon KD, Myers AJ, Craig DW, Webster JA, Pearson JV, et al. (2007) A high-density whole-genome association study reveals that APOE is the major susceptibility gene for sporadic late-onset Alzheimer's disease. J Clin Psychiatry 68: 613-618.

5. Profenno LA, Porsteinsson AP, Faraone SV (2010) Meta-analysis of Alzheimer's disease risk with obesity, diabetes, and related disorders. Biol Psychiatry 67: 505-512. [Crossref]

6. Fleminger S, Oliver DL, Lovestone S, Rabe-Hesketh S, Giora A(2003) Head injury as a risk factor for Alzheimer's disease: the evidence 10 years on; a partial replication. Journal of neurology, neurosurgery, and psychiatry 74: 857-862.

7. Letenneur L, Pérès K, Fleury H, Garrigue I, Barberger-Gateau P, et al. (2008) Seropositivity to herpes simplex virus antibodies and risk of Alzheimer's disease: a population-based cohort study. PLoS One 3: e3637.

8. Carter CJ (2010)Alzheimer's disease: a pathogenetic autoimmune disorder caused by herpes simplex in a gene-dependent manner. Int J AlzheimersDis201:140539.

9. Togo T, Akiyama H, Iseki E, Uchikado H, Kondo H, et al. (2004) Immunohistochemical study of tau accumulation in early stages of Alzheimer-type neurofibrillary lesions. Acta Neuropathol 107: 504-508.[Crossref]

10. Zhang YW, Thompson R, Zhang H, Xu H (2011) APP processing in Alzheimer's disease. Mol Brain 4: 3.[Crossref]

11. Sikorska B, Liberski PP, Sobow T, Budka H, Ironside JW(2009) Ultrastructural study of florid plaques in variant Creutzfeldt-Jakob disease: a comparison with amyloid plaques in kuru, sporadic Creutzfeldt-Jakob disease and Gerstmann-Straussler-Scheinker disease. Neuropathol Appl Neurobiol 35: 46-59.

12. Holmes C, Butchart J (2011) Systemic inflammation and Alzheimer's disease BiochemSoc Trans 39: 898-901.[Crossref]

13. Bonotis K, Krikki E, Holeva V, Aggouridaki C, Costa V, et al. (2008) Systemic immune aberrations in Alzheimer's disease patients. J Neuroimmunol 193: 183-187.[Crossref]

14. Levin EC, Acharya NK, Han M, Zavareh SB, Sedeyn JC, et al. (2010) Brain-reactive autoantibodies are nearly ubiquitous in human sera and may be linked to pathology in the context of blood-brain barrier breakdown. Brain Res 1345: 221-232.[Crossref]

15. Brookmeyer R, Evans DA, Hebert L, Langa KM, Heeringa SG, et al. (2011) National estimates of the prevalence of Alzheimer's disease in the United States. Alzheimers Dement 7: 61-73.[Crossref]

16. Itzhaki RF, Wozniak MA, Appelt DM, Balin BJ (2004) Infiltration of the brain by pathogens causes Alzheimer's disease. Neurobiol Aging 25: 619-627.[Crossref]

17. Balin B (2005) The Pathogen Hypothesis - Challenging the Primacy of Genetics in Late-Onset Alzheimer Disease: Biomedical Research Forum, Alforum.

18. Miklossy J (2011) Emerging roles of pathogens in Alzheimer disease. Expert Rev Mol Med 13: e30.[Crossref]

19. Roubaud-Baudron C, Krolak-Salmon P, Quadrio I, Megraud F, Salles N (2012) Impact of chronic Helicobacter pylori infection on Alzheimer's disease: preliminary results. Neurobiology of aging 33:1009.e11-1009.e19.

20. Moreno B, Jukes JP, Vergara-Irigaray N, Errea O, Villoslada P, et al. (2011) Systemic inflammation induces axon injury during brain inflammation. Annals of neurology 70 : 932-42.

21. Natalwala A, Potluri R, Uppal H, Heun R (2008) Reasons for hospital admissions in dementia patients in Birmingham, UK, during 2002-2007. Dement Geriatr Cogn Disord 26: 499-505.[Crossref]

22. Kellett KA, Hooper NM (2009) Prion protein and Alzheimer disease. Prion 3: 190-194. [Crossref]

Copyright: (C2017 Asher E. This is an open-access article distributed under the terms of the Creative Commons Attribution License, which permits unrestricted use, distribution, and reproduction in any medium, provided the original author and source are credited. 